\title{
Effect of Run Ze Tonic in Mice with Kidney-Yin Deficiency
}

Shuo Liu' ${ }^{1}$, Si-yao Ma ${ }^{1}$, Wen-yi Zhu², Jia Liang ${ }^{3}$, Hong Meng ${ }^{2 *}$

${ }^{1}$ Research and Development of Healthy Food, Infinitus (China) Co., Ltd., Guangzhou, China

${ }^{2}$ Cosmetics Science and Technology, Beijing Technology and Business University, Beijing, China

${ }^{3}$ Traditional Chinese Medicine, Graduate School of Chinese Academy of Traditional Chinese Medicine, Beijing, China

\section{*Corresponding author: Hong Meng,}

Beijing Technology and Business University, No. 11/33, Fucheng Road, Haidian District, Beijing, China

Tel.: +861061374229

Email: menghong2000@163.com

Received September 25, 2020

Revised October 28, 2020

Accepted November 19, 2020

Published March 30, 2021

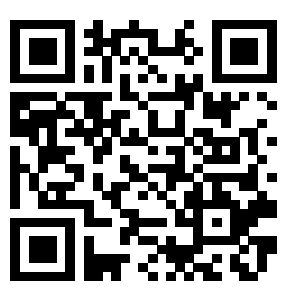

\begin{abstract}
Purpose: To observe the effects of Run Ze Tonic on thyroid hormone, adrenal function, sex hormones and cyclic nucleotide of mice with kidney-yin deficiency. Methods: ICR mice were randomly grouped into normal control group, model group, Run Ze Tonic high dose group, the middle dose group and low dose group, male and female in half. The kidney-yin deficiency mice models were established by intragastric administration of levothyroxine sodium before intragastric administration of corresponding drugs once a day for 10 consecutive days. One day after the last administration, the effects were observed by measuring weight. And tetraiodothyronine (T4), triiodothyronine (T3), thyrotropin (TSH), corticotropin releasing hormone $(\mathrm{CRH})$, adrenocorticotropic hormone (ACTH), corticosterone (CORT), testosterone (T), estradiol (E2), cyclic adenosine monophosphate (CAMP), cyclic guanosine monophosphate (cGMP) were tested by ELISA method. Results: Compared with the model group, the body weight, TSH level increased significantly $(p<0.01, p<0.05)$, and T3, T4, ACTH level decreased significantly in high dose group $(p<0.01, p<0.05)$. The E2 level in female decreased significantly in high dose group $(p<0.01)$, the same as the ratio of E2/T $(p<0.01)$. The T level in male increased significantly in high dose group $(p<0.01)$, the same as the ratio of E2/T $(p<0.01)$. And cAMP level decreased in the high dose group $(p<0.05)$. Conclusion: Run Ze Tonic may play the role of nourishing yin and strengthening kidney by adjusting hormone level disorder, adrenal cortex function and balancing energy metabolism in endocrine system.
\end{abstract}

Keywords: Run Ze Tonic, Kidney-yin deficiency, Adrenal function, Sex hormone, Cyclic nucleotide

\section{Introduction}

激肾阴虚证是指由于肾阴亏损，失于滋养，虚热内生所表现的 证候 (Wang \& Tian, 2008)。为了探索肾阴虚证的科学内涵, 采 用动物实验的方法进行研究 (Lin et al., 2013)。目前有多种肾 阴虚证动物模型用于功效评价, 其中甲状腺机能六进肾阴虚证模 型最为常用 (Wang \& Wang, 2013)。这种方法是通过给实验动物 使用甲状腺素, 形成外源性甲状腺素水平升高, 造成实验动物甲 状腺机能六进, 促进体内物质代谢, 增加耗氧量, 使产热增加, 能够模拟甲状腺功能立进时的消瘦、怕热、多汗、神经过敏、急 躁、震颤、心率加快、心输出量增加等症状, 表现基本符合临床 肾阴虚的辨证诊治（Zhang et al., 2015; Fu et al., 2017）。
润泽口服液由熟地黄、党参、桑椹、枸杞子、丹参、龙眼肉等 药食同源的中草药组成, 具有滋肾阴、补阴血的功效。因此, 本 实验采用甲状腺激素法建立肾阴虚证动物模型, 探讨润泽口服液 对肾阴虚动物模型的影响并阐释作用机制, 为滋阴补肾功效产品 的研制开发提供实验依据。

\section{Methods}

\section{Instruments and materials \\ 1) Test instrument}

全自动多功能酶标仪（MULTISKAN MK3; Thermofisher 
Scientific Co., LTD, The USA) ; 电热恒温培养箱 (DH4000A; Tianjin Taisite Instrument Co., LTD, China) ; 低温离心机 (5810R, Eppendorf AG, Germany) ; MINI shaker (MH-1; Haimen Kylin-Bell Lab Instruments Co., Ltd., China) 。

\section{2) Material}

润泽口服液（批号19C15BAB01; Infinitus（China）Co., Ltd., China）; 左甲状腺素钠（批号248922； Merk Serono GmbH, Germany) ; Mouse E2 elisa kit (CSB-E05109m; Cusabio Biotech C0., Ltd, China) ; Mouse T elisa kit (CSB-E05101m; Cusabio Biotech C0.); Mouse T3 elisa kit (CSB-E05086m; Cusabio Biotech C0. ) ; Mouse T4 elisa kit (CSB-E05083m; Cusabio Biotech C0.); Mouse TSH elisa kit (CSB-E05116m; Cusabio Biotech C0.); Mouse cAMP elisa kit (H164; Nanjing Jiancheng Bioengineering Institute, China); Mouse cGMP elisa kit (H163; Nanjing Jiancheng Bioengineering Institute)。

\section{3) Animal}

ICR小鼠 (SPF级), 雌雄兼用, 体重为 18-20 g, 购自北京华 阜康生物科技股份有限公司, 动物合格证号: 11401300086884, 使用许可证号：SCXK（京）2015-0041。

\section{Experimental method}

1) Animal grouping and treatment

将ICR 小鼠分为 5 组, 每组 20 只, 雌雄各半。正常组、模型组、 女仕牌固体饮料大 ( $1.65 \mathrm{~g} / \mathrm{kg} / \mathrm{d})$ )、中 ( $0.83 \mathrm{~g} / \mathrm{kg} / \mathrm{d})$ 、小剂量 组 $(0.41 \mathrm{~g} / \mathrm{kg} / \mathrm{d})$, 分别相当于成人日用量的 2 倍, 1 倍, 0.5 倍。除正常组外, 各组小鼠给予左甲状腺素钠 $40 \mu \mathrm{g} / \mathrm{kg} \mathrm{ig}, 0.1$ $\mathrm{mL} / 10 \mathrm{~g}$, 每天 1 次, 连续 10 天, 造成肾阴虚证候小鼠模型。 每日给予左甲状腺素钠后间隔 2 小时给予相应药物 $0.2 \mathrm{~mL} / 10 \mathrm{~g}$ $i g$, 连续 10 天, 正常组和模型组在同等条件下给予蒸馏水。

\section{2) Detection of index}

每天观察大鼠饮食饮水情况、精神状态、毛色、体态, 末次 给药后称取体质量。观察小鼠一般体征。第 11 天称重后眼眶
取血, 离心分离血清, $-20^{\circ} \mathrm{C}$ 保存待测, 采用ELISA方法检测甲状 腺素（thyroxine，T4）、三碘甲状原氨酸（triiodothyronine, T3）、促甲状腺激素（thyrotropin，TSH）、睪酮（testosterone, $\mathrm{T}$ ）、雌二醇（estradio1, E2）、促肾上腺激素释放激素 (corticotropin releasing hormone, CRH) 、促肾上腺皮 质激素（adrenocorticotropic hormone, ACTH）、皮质酮 (corticosterone, CORT), 环磷酸腺苷 (cyclic Adenosine monophosphate, cAMP) 、环磷酸鸟苷 (cyclic guanosine monophosphate, cGMP) 的表达。解剖, 取小鼠脾脏、胸腺、肾上 腺, 称重, 计算脾指数、胸腺指数和肾上腺指数。

脾指数 $(\%)=[$ 脾湿重 $(\mathrm{g}) /$ 体重 $(\mathrm{g})] \times 100$

胸腺指数 $(\%)=[$ 胸腺湿重 $(\mathrm{g}) /$ 体重 $(\mathrm{g})] \times 100$

肾上腺指数 $(\%)=[$ 肾上腺湿重 $(\mathrm{g}) /$ 体重 $(\mathrm{g})] \times 100$

\section{Statistical analysis}

各组所得数据均以 $(M \pm s)$ 表示, 组间比较采用 $t$-test检验。

\section{Results}

\section{Effect of sample on general physical signs in mice with kidney-yin deficiency}

正常组小鼠毛发润泽、活动自如; 连续给予左甲状腺素钠 10 天后, 模型组小鼠出现躁动、易惊、体毛粗糙无光泽等表现, 体重增长缓慢, 与正常组比较有极显著性差异 $(p<0.01)$ 。灌胃 给予润泽口服液后小鼠毛色和光泽度有所改善, 易惊表现有改 善趋势。无限极牌润泽口服液大剂量小鼠体重增加, 与模型组 比较均有显著性差异 $(p<0.05)$ ，无限极牌润泽口服液中剂量和 小剂量组小鼠体重没有明显改变, 与模型组比较无显著性差异 $(p>0.05)$ 。见Table 1 。

\section{Effect of sample on organ index in mice with kidney-yin deficiency}

给予左甲状腺素钠后, 模型组小鼠脾指数升高, 与正常组比较 有显著性差异 $(p<0.05)$ ，胸腺指数和肾上腺指数未见显著改变

Table 1. Effect of sample on body mass in mice with kidney-yin deficiency

\begin{tabular}{lcc}
\hline Group & Before model establishment & After model establishment \\
Normal group & - & $28.83 \pm 3.79^{1)}$ \\
Model group & $11 \mathrm{~mL} / \mathrm{kg} / \mathrm{d}$ & $25.52 \pm 1.98^{\# \#}$ \\
High dose group & $5.5 \mathrm{~mL} / \mathrm{kg} / \mathrm{d}$ & $27.20 \pm 2.92^{*}$ \\
Middle dose group & $2.75 \mathrm{~mL} / \mathrm{kg} / \mathrm{d}$ & $26.53 \pm 2.35$ \\
Low dose group & 2.09 \\
\hline
\end{tabular}

${ }^{1)}$ Mean \pm standard deviation; Compared with normal group, ${ }^{\# \#} p<0.01$; Compared with model group, ${ }^{*} p<0.05$. 
$(p>0.05)$; 灌胃给予润泽口服液后脾指数、胸腺指数、肾上腺 指数均无显著改变, 与模型组比较均无显著性差异 $(p>0.05)$ 。 见Table 2。

3. Effect of sample on hyperthyroidism in mice with kidneyyin deficiency

给予左甲状腺素钠后, 模型组小鼠血清TSH含量降低, T3、T4含 量升高, 与正常组比较均有显著性差异 $(p<0.01)$; 润泽口服液 大剂量大剂量组血清TSH含量升高, 与模型组比较有显著性差异 $(p<0.01)$; 润泽口服液大剂量组血清T3、T4含量均降低，与模 型组比较有显著性差异 $(p<0.01)$ 。见Table 3 。

4. Effect of sample on adrenal function in mice with kidneyyin deficiency

给予左甲状腺素钠后, 模型组小鼠血清ACTH含量升高, 与正常 组比较均有显著性差异 $(p<0.01)$; 模型组小鼠血清CRH和CORT含
量无显著变化, 与正常组比较均无显著性差异 $(p>0.05)$ 。给予 受试样品后, 润泽口服液大剂量组血清ACTH含量降低, 与模型组 比较有显著性差异 $(p<0.05)$; 中剂量组和小剂量组ACTH含量没有 显著变化，与模型组比较无显著性差异 $(p>0.05)$ 。见Table 4 。

5. Effect of sample on the level of sex hormone in mice with kidney-yin deficiency

对于雌性小鼠：模型组血清E2含量升高, T含量降低, E2/T升 高, 与正常组比较均有显著性差异 $(p<0.01, p<0.05)$; 给予润 泽口服液后, 大剂量组雌鼠 $\mathrm{E} 2$ 含量降低, 大剂量组雌鼠 $\mathrm{E} 2 / \mathrm{T}$ 比值 降低，与模型组比较均有显著性差异 $(p<0.05)$ 。

对于雄性小鼠: 模型组血清T含量降低, E2/T升高, 与正常组比 较均有显著性差异 $(p<0.05)$, 模型组血清E2含量无显著改变, 与正常组比较均无显著性差异（ $p>0.05 ）$; 给予润泽口服液后， 大剂量雄鼠血清E2/T有降低趋势, 但与模型组比较无显著性差异 $(p>0.05)$ 。见Table 5。

Table 2. Effect of sample on organ index in mice with kidney-yin deficiency

$(\mathrm{N}=\mathbf{2 0})$

\begin{tabular}{lccc}
\hline Group & Spleen index & Thymic index & Adrenal index \\
Normal group & $0.47 \pm 0.05^{1)}$ & $0.38 \pm 0.06$ & $0.04 \pm 0.03$ \\
Model group & $0.51 \pm 0.05^{\#}$ & $0.36 \pm 0.09$ & $0.04 \pm 0.02$ \\
High dose group & $0.52 \pm 0.10$ & $0.34 \pm 0.07$ & $0.04 \pm 0.02$ \\
Middle dose group & $0.54 \pm 0.11$ & $0.37 \pm 0.11$ & $0.04 \pm 0.01$ \\
Low dose group & $0.54 \pm 0.09$ & $0.36 \pm 0.07$ & $0.04 \pm 0.01$ \\
\hline
\end{tabular}

${ }^{1)}$ Mean \pm standard deviation; Compared with normal group, ${ }^{\sharp} p<0.05$.

Table 3. Effect of sample on serum TSH, T3, T4 level in in mice with kidney-yin deficiency

$(\mathrm{N}=\mathbf{2 0})$

\begin{tabular}{llll}
\hline Group & TSH $(\mathrm{ulU} / \mathrm{mL})$ & $\mathrm{T} 3(\mathrm{ng} / \mathrm{mL})$ & $\mathrm{T} 4(\mathrm{ng} / \mathrm{mL})$ \\
Normal group & $6.08 \pm 0.76$ & $3.96 \pm 0.52$ & $211.72 \pm 42.75$ \\
Model group & $1.47 \pm 0.20^{\# \#}$ & $5.84 \pm 0.81^{\# \#}$ & $536.88 \pm 63.45^{\# \#}$ \\
High dose group & $2.33 \pm 0.60^{* *}$ & $4.64 \pm 0.63^{* *}$ & $410.53 \pm 58.76^{* *}$ \\
Middle dose group & $1.89 \pm 0.52$ & $5.62 \pm 0.73$ & $489.38 \pm 90.21$ \\
Low dose group & $2.02 \pm 0.82$ & $5.41 \pm 0.65$ & $483.52 \pm 83.20$ \\
\hline
\end{tabular}

TSH, thyropin; T3, triiodothyronine; T4, tetraiodothyronine; ${ }^{1)}$ Mean \pm standard deviation; Compared with normal group, ${ }^{\# \#} p<0.01$; Compared with model group, ${ }^{* *} p<0.01$.

Table 4. Effect of sample on serum CRH, CORT, ACTH in mice with kidney-yin deficiency

$(\mathbf{N}=\mathbf{2 0})$

\begin{tabular}{llcc}
\hline Group & $\mathrm{CRH}(\mathrm{ng} / \mathrm{mL})$ & $\mathrm{CORT}(\mathrm{ng} / \mathrm{mL})$ & $\mathrm{ACTH}(\mathrm{pg} / \mathrm{mL})$ \\
Normal group & $41.76 \pm 8.35^{1)}$ & $1692.47 \pm 215.31$ & $923.45 \pm 98.22$ \\
Model group & $47.58 \pm 7.29$ & $1539.02 \pm 223.56$ & $1121.45 \pm 90.45^{\# \#}$ \\
High dose group & $42.49 \pm 10.34$ & $1503.22 \pm 163.35$ & $971.26 \pm 88.23^{*}$ \\
Middle dose group & $48.43 \pm 9.79$ & $1487.03 \pm 194.32$ & $1133.21 \pm 230.88$ \\
Low dose group & $47.16 \pm 12.77$ & $1426.37 \pm 158.73$ & $1092.54 \pm 213.55$ \\
\hline
\end{tabular}

$\mathrm{CRH}$, corticotropin releasing hormone; CORT, corticosterone, ACTH, adrenocorticotropic hormone; ${ }^{1}$ Mean \pm standard deviation; Compared with normal group, ${ }^{\# \#} p<0.01$; Compared with model group, ${ }^{*} p<0.05$. 
6. Effect of sample on cyclic nucleotide level in mice with kidney-yin deficiency

模型组小鼠血清CAMP含量升高, cAMP/cGMP升高, 与正常组比 较均有显著性差异 $(p<0.05), \mathrm{CGMP}$ 无显著变化 $(p>0.05)$; 给 予女仕牌固体饮料后, 各剂量组小鼠血清CAMP含量降低, 与模 型组比较均有显著性差异 $(p<0.05)$; 女仕牌固体饮料各剂量 组CGMP含量, 与模型组比较均无显著差异 $(p>0.05)$; 女仕牌固 体饮料小剂量组 $\mathrm{cAMP} / \mathrm{cGMP}$ 降低, 与模型组比较均有显著性差异 $(p<0.05)$ 。见Table 6。

\section{Discussion}

肾阴虚是中医学虚证的基本证型之一, 是指由于肾阴亏损, 失于滋养, 虚热内生所表现的证候, 肾阴虚证见腰膝酸痛, 失眠 多梦, 头晕耳鸣, 咽干歡红, 五心烦热, 遗精早泄, 潮热盗汗, 舌红少津无苔, 脉细数等 (Fu et al., 2017)。临床肾阴虚患者 多见体形消瘦、皮肤干燥、心烦易怒, 模型组小鼠体重减轻一定 程度上说明虚损的程度。肾阴虚主要与甲状腺轴、性腺轴、能量 代谢、免疫力下降等有关 (Fu et al., 2017; Dai et al., 2016; Zou et al., 2015)。中医认为, 甲状腺机能六进属阴虚证范畴 (Huang et al., 2008), T3、T4升高多见于五心烦热, 咽干口燥, 舌红少苔, 脉细数的肾阴虚型患者 (Fang, 2008)。甲状腺功能 六进可出现血清 $\mathrm{T} 3 、 \mathrm{~T} 4$ 含量明显升高, 基础代谢增强（Huang et al. ， 2011），负反馈抑制腺垂体分泌TSH，二者互相拮抗，T3、 T4、TSH表达异常能反映甲状腺轴功能紊乱 (Dai et al., 2016)。 在临床检查中, 性激素E2、T和E2/T比值异常能反映性腺轴功能紊 乱, 是阴阳失调的重要指标 (Xu et al., 2007)。但各类肾虚类型 的T与E2水平及变化不尽相同（Qiu et al., 1999)。有研究将E2含 量上升、T含量下降, E2/T作为肾阴虚大鼠模型造模成功的评判标 准(Peng \& Zhang., 2003)。环核苷酸cAVP、cGMP是调节细胞功能活 动的重要物质, 二者相互制约、相互拮抗, 其对生物细胞的双向 调节作用与中医阴阳学说相似 (Cheng, 2016; Li et al., 2017)。 $\mathrm{cAMP} / \mathrm{cGMP}$ 升高可反映肾阴虚动物模型造模成功。本实验中, 左 甲状腺素钠诱导肾阴虚模型小鼠出现躁动、易惊、体毛粗粘无光 泽, 体重增长缓慢等状态, 表现虚损症状。肾阴虚小鼠血清T3、 T4含量上升, TSH含量下降, ACTH 含量升高, T含量下降、E2/T比 值上升, cAMP / cGMP比值升高, 均表明肾阴虚模型造模成功。

润泽口服液由熟地黄、党参、桑椹、枸杞子、丹参、龙眼肉等 药食同源的中草药组成, 本研究采用左甲状腺素钠诱导肾阴虚模 型评价润泽口服液干预肾阴虚的作用, 分为大、中、小剂量组, 在造模后给予润泽口服液干预, 不仅观察润泽口服液干预肾阴虚 的功效, 同时初步探讨对肾阴虚的作用机制。

机体处于甲方状态与下丘脑-垂体一甲状腺轴（HPT）相关（Duan et al.，2016), 甲六型肾阴虚小鼠的甲状腺功能六进、基础代谢 增多, 躁动、易惊扰, 体毛干枯、体重下降, 血清 $\mathrm{T} 3$, T4 含量升 高, TSH含量降低。本实验结果显示, 大剂量润泽口服液可改善 肾阴虚模型动物毛色和光泽度, 在一定程度上改善模型动物的易

Table 5. Effect of sample on serum E2, $\mathrm{T}$ level and ratio of E2/T in mice with kidney-yin deficiency

$\mathbf{( N = 2 0 )}$

\begin{tabular}{|c|c|c|c|c|c|c|}
\hline \multirow{2}{*}{ Group } & \multicolumn{2}{|c|}{$\mathrm{E} 2(\mathrm{pg} / \mathrm{mL})$} & \multicolumn{2}{|c|}{$\mathrm{T}(\mathrm{pg} / \mathrm{mL})$} & \multicolumn{2}{|c|}{$\mathrm{E} 2 / \mathrm{T}$} \\
\hline & Female & Male & Female & Male & Female & Male \\
\hline Normal group & $183.91 \pm 8.80^{1)}$ & $175.47 \pm 24.22$ & $0.84 \pm 0.08$ & $2.85 \pm 0.40$ & $222.31 \pm 29.31$ & $62.58 \pm 11.96$ \\
\hline Model group & $223.80 \pm 21.98^{\# \#}$ & $168.81 \pm 10.68$ & $0.68 \pm 0.07^{\# \#}$ & $2.05 \pm 0.84^{\#}$ & $332.41 \pm 57.60^{\# \#}$ & $92.32 \pm 31.20^{\#}$ \\
\hline High dose group & $190.03 \pm 21.76^{* *}$ & $166.66 \pm 36.48$ & $0.72 \pm 0.09$ & $2.74 \pm 0.23^{*}$ & $265.98 \pm 32.26^{* *}$ & $61.07 \pm 13.98^{*}$ \\
\hline Middle dose group & $204.73 \pm 24.78$ & $177.11 \pm 24.99$ & $0.62 \pm 0.10$ & $2.21 \pm 0.59$ & $338.09 \pm 63.95$ & $85.69 \pm 25.35$ \\
\hline Low dose group & $215.66 \pm 10.79$ & $163.30 \pm 10.07$ & $0.63 \pm 0.06$ & $2.29 \pm 0.59$ & $346.07 \pm 25.32$ & $75.27 \pm 17.76$ \\
\hline
\end{tabular}

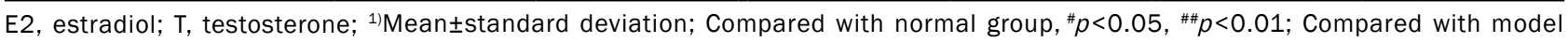
group, ${ }^{*} p<0.05,{ }^{* *} p<0.01$.

Table 6. Effect of sample on serum cAMP and cGMP level and the ratio of cAMP/cGMP in mice with kidney-yin deficiency (N=20)

\begin{tabular}{lllc}
\hline Group & cAMP $(\mathrm{nmol} / \mathrm{L})$ & $\mathrm{cGMP}(\mathrm{nmol} / \mathrm{L})$ & cAMP/cGMP \\
Normal group & $141.15 \pm 20.82^{1)}$ & $162.45 \pm 24.26$ & $0.89 \pm 0.17$ \\
Model group & $159.85 \pm 15.20^{\# \#}$ & $147.25 \pm 29.81$ & $1.13 \pm 0.26^{\# \#}$ \\
High dose group & $145.58 \pm 15.45^{* *}$ & $144.59 \pm 20.38$ & $1.03 \pm 0.18$ \\
Middle dose group & $150.24 \pm 21.25$ & $153.29 \pm 31.21$ & $1.01 \pm 0.21$ \\
Low dose group & $157.14 \pm 15.28$ & $152.52 \pm 12.44$ & $1.04 \pm 0.12$ \\
\hline
\end{tabular}

cAMP, cyclic adenosine monophosphate; cGMP, cyclic guanosine monophosphate; ${ }^{11}$ Mean \pm standard deviation; Compared with normal group, ${ }^{\sharp} p<0.05$, ${ }_{\# \#} p<0.01$; Compared with model group, ${ }^{* *} p<0.01$. 
惊状态, 并可增加模型动物体重, 具有降低肾阴虚动物模型血清 T3、T 、 升高TSH作用。研究从分子水平提示, 润泽口服液能改善 甲状腺功能亢进, 改善HPT轴功能。

$H P A$ 轴是由下丘脑、垂体与肾上腺组成的维持人体基本生命活 动的重要神经内分泌轴之一, 下丘脑室旁核分泌促肾上腺皮质激 素释放激素 (CRH) , 经垂体门脉系统到达腺垂体, 与促肾上腺皮 质激素 (ACTH) 细胞膜上的CRH受体结合, 促进腺垂体分泌ACTH并 作用与肾上腺皮质细胞, 促进肾上腺皮质的增生, 并促进束状带 合成和分泌糖皮质激素 (Cor.) , 参与机体的代谢、免疫以及生 长发育过程, 对于维持机体的内稳态起着重要作用（Xu et al., 2009）。内分泌系统功能障碍是虚证的重要表现之一, 肾阴虚患者 常常表现为HPA轴功能六进, 即表现为下丘脑分泌CRH、垂体分泌 ACTH增加，进而刺激肾上腺皮质释放Cor.，使血中Cor. 水平明显升 高 (Xia et al., 2013)。对于啮齿类动物, 体内皮质酮（CORT） 含量可代表Cor. 水平。本实验结果显示, 大剂量润泽口服液可降 低肾阴虚小鼠血清ACTH含量。研究提示润泽口服液通过降低HPA轴 中分泌的ACTH含量, 改善肾上腺皮质系统功能, 进而改善肾阴虚 证。

中医认为 “肾藏精, 主生长发育与生殖”, 肾气的盛衰直接影 响着人体的生殖功能, 而现代医学也发现生殖系统的功能受下丘 脑-垂体-性腺 (HPG) 轴神经内分泌调节, 肾阴虚与HPG轴功能密切 相关 (Zhang et al., 2017)。本实验结果显示女仕牌固体饮料可降 低雌性肾阴虚动物模型血清E2含量, 降低E2/T比值, 升高雄性肾阴 虚动物模型血清 $T$ 含量、降低 $\mathrm{E} 2 / \mathrm{T}$ 比值, 研究提示润泽口服液能在 一定程度上调节性激素平衡, 改善HPG轴功能。

机体处于正常状态下, 血清的cAMP, cGIP水平不会发生变化, 一 旦机体持续处于六奋状态, 通过激活腺苷酸环化酶的活性, 诱导 cAVP, cGMP 的合成。CAMP 和CGMP是调节物质代谢和生物学功能的 重要物质, 对细胞功能处于稳定状态具有双向控制调节作用（Pan et al., 2014), 且与中医学的阴阳学说有相似之处, 研究表明, cAMP含量升高是肾阴虚的特征之一 (Chen，2011), 本实验结果显 示大剂量润泽口服液可降低肾阴虚动物模型血清CAVP含量, 降低 cAVP/ cGMP比值, 研究提示润泽口服液在一定程度上具有平衡环核 苷酸系统的作用。

\section{Conclusion}

润泽口服液能在一定程度上改善肾阴虚小鼠虚损状态，调节肾 阴虚证的相关物质基础促甲状腺激素（TSH）、三碘甲状腺原氨 酸（T3）、甲状腺素（T4）、促肾上腺皮质激素 (ACTH)、雌二醇 （E2）、睪酮（T）、环磷酸腺苷（cAMP）水平，从而改善甲状腺 功能进, 改善肾上腺皮质功能, 平衡性激素及环核苷酸系统。 润泽口服液可能通过调整内分泌系统中激素水平紊乱、调节能量
代谢，发挥滋阴补肾功效。

\section{Author's contribution}

SL and HM designed the experiment, and WYZ and JL operated the animal experiment. SL and SYM analyzed the data, and HM wrote the article.

\section{Author details}

Shuo Liu (Engineer), Research and Development of Healthy Food, Infinitus (China) Co., Ltd., Infinitus R\&D center, Hongtai Wisdom Valley, 19th of Si Cheng Road, Tian He District, Guangzhou 510663, China; Si-yao Ma (Engineer), Research and Development of Healthy Food, Infinitus (China) Co., Ltd., Infinitus R\&D center, Hongtai Wisdom Valley, 19th of Si Cheng Road, Tian He District, Guangzhou 510663, China; Wen-yi Zhu (Graduate student), College of Chemistry and Materials Engineering, Beijing Technology and Business University, 11 Fucheng Road, Haidian District, Beijing 10048, China; Jia Liang (Graduate student), Traditional Chinese Medicine, Graduate School of Chinese Academy of Traditional Chinese Medicine, 16 Dongzhimen neinan street, Dongcheng District, Beijing 100700, China; Hong Meng (Professor), College of Chemistry and Materials Engineering, Beijing Technology and Business University, 11 Fucheng Road, Haidian District, Beijing 10048, China.

\section{References}

Cheng SK. The combination of traditional Chinese and western medicine and Its enlightenment (3)-professor Kuang Ankun opened up a "ice breaking trip" for the study of "Yin Yang theory" of traditional Chinese Medicine. Chinese Journal of Integrated Traditional and Western Medicine, 36: 1285-1289, 2016.

Chen Q. Research methods in pharmacology of Chinese materia medica. People's Medical Publishing House, Beijing, p1028, 2011.

Duan S, Chen J, Tan W, Liang J, Yan YY. Correlation between adrenal cortical function and kidney deficiency syndrome in autoimmune thyroid disease. Chinese Journal of Integrative Medicine on Cardio-/Cerebrovascuiar Disease, 14: 2227-2229, 2016. 
Dai B, Yang ML, Zhang JN, Li YX, Ouyang LQ, Xiao ZZ, Deng JM, Yang L. Effect of Liuweidihuang decoction and "Yibu Yixie" on HPT Axis in kidney-yin deficiency mice model. Information on Traditional Chinese Medicine, 33: 19-22, 2016.

Fang ZQ. Syndrome differentiation and treatment of traditional Chinese medicine. Shanghai University of Traditional Chinese Medicine Press, Shanghai, p566, 2008.

Fu HE, Li JM, Liu YH. Effect of Zuoguiwan on neuro-endocrineimmune function of kidney-yin deficiency rats. Chinese Journal of Experimental Traditional Medical Formulae, 23: 155-159, 2017.

Huang DB, Hu XG, Pu GX. Pharmacology and clinic of Integrated Chinese and Western Medicine. Hubei Science \& Technology Press, Wuhan, p434, 2008.

Huang J, Li X, Zhang J, Du Y, Zhang Y. Effect of Liuwei Dihuang Wan on Shenyin vacuity model mice with hyperthyroidism by thyroxin and reserpine. Pharmacology and Clinics of Chinese Materia Medica, 27: 1-3, 2011.

Li YJ, Wei DD, Wei ML, Wang XK, Zhang Y, Yue XX, Ding PN, Yang $X Y$, Cui LH. Evaluation of three kinds of kidney yin deficiency modeling based on animal substance and energy metabolism detection. Chinese Journal of Chinese Medicine, 32: 83-88, 2017.

Lin ZJ, Zhang B, Liu XQ, Zhu WJ. Research on animal model evaluation with traditional Chinese medicine principles. China Journal of Traditional Chinese Medicine and Pharmacy, 28: 2217-2221, 2013.

Pan X, Hu CJ, Geng YY, Zhao L, Xiong R, Chen ZM. T lymphocyte sub-population and CAMP/cGMP regulating effect by processed ershen pill formula. Chinese Journal of Experimental Traditional Medical Formulae, 13: 138141, 2014.

Peng Y, Zhang YD. Research status of kidney-deficiency and sex hormone. Heilongjiang Journal of Traditional Chinese Medicine, 6: 50-51, 2003.
Qiu RX, Wu GZ, Jin MH. Regulating effect of sex hormone on the balance of patients with kidney-yin and kidneyyang deficiency. Chinese Journal of Basic Medicine in Traditional Chinese Medicine, 3: 46-47, 1999.

Wang D, Tian FH. Research on kidney-yin deficiency. Journal of Liaoning University of TCM, 10: 33-35, 2008.

Wang P, Wang XJ. Research overview of animal model with kidney-yin deficiency. Information on Traditional Chinese Medicine, 30: 123-125, 2013.

Xia BJ, Tong PJ, Sun Y, Zhou LY, Jin HT. Research on osteoporosis mice model with type of deficiency of kidney-yin. Journal of Emergency in Traditional Chinese Medicine, 22: 1083-1086, 2013.

Xu CP, Sun J, Zhu QJ, Song J, Zhang D, Li Z. Effect of Jinkui Shenqi pill on hypothalamus pituitary adrenal axis function of kidney yang deficiency model mice. Journal of Shandong University of TCM, 33: 248-249, 2009.

Xu XQ, Huang JY, Luo R, Xiao BQ, Zhao T, Tu XY. Effect of active fraction in Erjing pill on learning dysmnesia of rat with kidney-yin deficiency and its molecular mechanism. Chinese Traditional and Herbal Drugs, 38: 564-569, 2007.

Zhang JN, Li YX, Yang ML, Xiao ZZ, Dai B, Xiao WZ, Ouyang LQ. Effects of Liuwei Dihuang decoction and "reinforcing and reducing" herb couples on HPG axis in kidney Yin deficiency mice. Chinese Journal of Modern Applied Pharmacy, 34: 25-29, 2017.

Zhang ZY, Chen BJ, Zhang YJ, Wang WT, Wang YR, Su YX. Establishment and stability observation of rat model with kidney-yang deficiency and kidney-yin deficiency. Fujian Journal of TCM, 46: 51-54, 2015.

Zou HM, Zhang B, Sun W, Zhao L, Yang YT, Zhao AH, Qiu MF. Research progress in biochemical indicators of syndrome of deficiency of kidney yin. China Journal of Traditional Chinese Medicine and Pharmacy, 30: 36073610, 2015. 


\section{中文摘要}

\section{润泽口服液对肾阴虚小鼠的影响研究}

刘硕 ${ }^{1}$, 马思遥 ${ }^{1}$, 朱文驿 $^{2}$, 梁佳 $^{3}$, 孟宏 $^{2 *}$

${ }^{1}$ 无限极（中国）有限公司健康食品研发，广州，中国

${ }^{2}$ 北京工商大学化妆品科学与技术, 北京, 中国

${ }^{3}$ 中国中医科学院研究生院中医学, 北京, 中国

目的: 从甲状腺功能、肾上腺皮质功能、性激素水平、环核苷酸系统等方面探讨润泽口服液对肾阴虚小鼠模型 的影响。方法: ICR 小鼠随机分为正常组、模型组、润泽口服液大、中、小剂量组 $(1.65,0.83,0.41 \mathrm{~g} / \mathrm{kg} /$ d），每组 20 只。灌胃给予 $40 \mu \mathrm{g} / \mathrm{kg}$ 左甲状腺素钠，连续 10 天，造成肾阴虚证候小鼠模型。观察各组小鼠一般 体征、体重, 计算脏器指数, 检测血清甲状腺素（T4）、三碘甲状原氨酸（T3）、促甲状腺激素（TSH）、促 肾上腺激素释放激素 $(\mathrm{CRH})$ 、促肾上腺皮质激素 $(A C T H)$ 、皮质酮 $(C O R T)$ 、睪酮 $(T)$ 、雌二醇（E2）、环 磷酸腺苷 (cAMP) 、环磷酸鸟苷 (cGMP) 的表达。结果: 大剂量润泽口服液可以改善肾阴虚小鼠动物一般体 征, 增加体重 $(p<0.05)$ ，降低肾阴虚小鼠血清T3、T4含量、升高TSH含量 $(p<0.01)$; 降低肾阴虚动物模型 血清ACTH水平 $(p<0.05)$; 降低肾阴虚雌小鼠血清中性激素 E2 含量及E2/T比值 $(p<0.01)$ ，升高肾阴虚雄小 鼠血清中性激素 T 含量 $(p<0.05)$; 降低环核苷酸系统CAMP含量 $(p<0.01)$ 。结论: 润泽口服液通过调整内分 泌系统中激素水平紊乱、肾上腺皮质功能、平衡调节能量代谢，发挥滋阴补肾功效。

关键词：润泽口服液，肾阴虚，肾上腺皮质功能，性激素，环核苷酸系统 


\section{국문초록}

\section{Run Ze Tonic의 신장-음 결핍 마우스에 대한 효능}

류석 ${ }^{1}$, 마사요 ${ }^{1}$, 주문이 ${ }^{2}$, 양가 ${ }^{3}$, 맹홍 ${ }^{2 *}$

${ }^{1}$ 무한급(중국)유한회사 건강식품개발, 광주, 중국

${ }^{2}$ 북경공상대학 화장품과학과 기술, 북경, 중국

중국중의과학원연구생원 중의학, 북경, 중국

목적: 본 연구는 Run Ze Tonic의 신장-음 결핍 마우스의 갑상선 호르몬, 부신 기능, 성 호르몬 및 주기적 뉴클레오티드에 미치는 영 향을 관찰하고자 한다. 방법: ICR 마우스를 정상 대조군, 모델 그룹, Run Ze Tonic 고용량 그룹, 중간 용량 그룹 및 저용량 그룹으로 분류하였으며, 매 그룹은 20 마리씩 구성하였다. 신장-음 결핍 마우스 모델은 연속 10 일 동안 1 일 1 회 레보 티록신 나트륨을 위내 직접 투여에 의해 확립되었다. 각 그룹의 마우스의 일반특징 및 체중을 관찰하고 장기지수를 측정하였다. 그리고 ELISA 방법으로 테트라 요오 도티 로닌 (T4), 트리 요오 도티 로닌 (T3), 갑상선 호르몬 (TSH), 코르티코 트로 핀 방출 호르몬 (CRH), 부 신피질 자극 호르몬 (ACTH), 코르티 코스 테론 (CORT), 테스토스테론 (T), 에스트라 디올 (E2), 고리 형 아데노신 모노 포스페이트 (cAMP), 사 이 클릭 구아노 신 모노 포스페이트 (cGMP)를 측정하였다. 결과: Run Ze Tonic 고용량군은 신장-음결핍 마우스의 일반적인 신체 징후를 개선하고, 체중을 증가시키고 $(p<0.05), \mathrm{TSH}$ 함량을 증가시킨다 $(p<0.01)$. 고용량 군에서 T3 및 T4 수치가 유의하게 감소하 였으며 $(p<0.01), \mathrm{ACTH}$ 수준도 유의하게 감소하였다 $(p<0.05)$. 신장 음 결핍 암컷 마우스는 고용량 군에서 성 호르몬 $\mathrm{E} 2$ 및 $\mathrm{E} 2 / \mathrm{T}$ 비 율이 유의하게 감소 $(p<0.01)$ 하였다. 신장 음이 결핍 된 수컷 마우스의 경우 고용량 군에서 성 호르몬 T함량이 유의하게 증가되었다 $(p<0.05)$. 그리고 고용량 군에서 고리 형 뉴클레오티드 시스템의 cAMP 수치가 감소하였다 $(p<0.01)$. 결론: Run Ze Tonic은 내분비 계에서 호르몬 수치 장애, 부신 피질 기능 및 에너지 대사 균형을 조절하여 음양을 키우고 신장을 강화하는 역할을 할 수 있다고 사 료된다.

핵심어: Run Ze Tonic, 신장 결핍, 부신 기능, 성 호르몬, 주기적 뉴클레오타이드 